\title{
Lung transplantation for patients with severe COVID-19-related acute respiratory distress syndrome in Korea
}

\author{
Ryoung-Eun Ko ${ }^{1}$, Dong Kyu Oh², Sun Mi Choi ${ }^{3}$, Sunghoon Park ${ }^{4}$, Ji Eun Park ${ }^{5}$, Jin Gu Lee ${ }^{6}$, Young Tae Kim \\ Kyeongman Jeon ${ }^{8}$

\footnotetext{
${ }^{1}$ Department of Critical Care Medicine, Samsung Medical Center, Seoul, Korea

${ }^{2}$ Department of Pulmonary and Critical Care Medicine, Asan Medical Center, University of Ulsan College of Medicine, Seoul, Korea

${ }^{3}$ Department of Pulmonary and Critical Care Medicine, Seoul National University Hospital, Seoul, Korea

${ }^{4}$ Department of Pulmonary, Allergy and Critical Care Medicine, Hallym University Sacred Heart Hospital, Anyang, Korea

${ }^{5}$ Department of Pulmonary and Critical Care Medicine, Ajou University Hospital, Suwon, Korea

${ }^{6}$ Department of Thoracic and Cardiovascular Surgery, Severance Hospital, Seoul, Korea

${ }^{7}$ Department of Thoracic and Cardiovascular Surgery, Seoul National University Hospital, Seoul, Korea

${ }^{8}$ Department of Pulmonary and Critical Care Medicine, Samsung Medical Center, Seoul, Korea
}

Background: There are uncertainty of lung transplantation (LT) in patients with COVID-19-related acute respiratory distress syndrome (ARDS) who failed to recovery despite optimal management including extracorporeal membrane oxygenation (ECMO).

Methods: Nationwide multi-center retrospective observational study was performed with consecutive lung transplants for severe COVID-19-related ARDS in South Korea between June 2020 and June 2021. Data on patient demographics, pre-transplant and perioperative characteristics, and post-transplant outcomes were collected and compared with other LTs with ECMO bridge from the Korean Organ Transplantation Registry.

Results: A total of 11 patients with COVID-19-related ARDS underwent LT at the five centers in South Korea. The median age was 60.0 years (interquartile range [IQR], 57.5-62.5); six were male. At listing, all patients were supported with veno-venous ECMO. The median clinical frailty scale was $1.0(\mathrm{IQR}, 1.0-2.0)$ and three patients $(27.3 \%)$ were on renal replacement therapy. All patients received rehabilitation for the median of 28.0 (IQR, 17.5-43.0) days before LT. Patients were transplanted a median of 49 days (IQR, 32-66) after ECMO cannulation. Primary graft dysfunction (PGD) within 72 hours of LT was developed in two patients (18.2\%). Major postoperative complications were infection in seven (63.5\%) and bleeding requiring interventions in four (36.4\%). One patient died 4 days after LT due to sepsis and one patient underwent re-transplant for graft failure. After a median follow up of 112 days (IQR, 97-166), 10 patients are alive and recovering well. Compared to other LTs with ECMO bridge $(n=27)$, post-transplant outcomes including PGD and mortality were not different between the two groups. However, infection was more frequent in patients with COVID-19-related ARDS (63.6\% vs. $14.8 \%$; $P=0.005)$.

Conclusions: LTs in patients with unresolving COVID-19-related ARDS were effective with reasonable short-term outcomes, which was similar to other LTs with ECMO bridge.

Corresponding author: Kyeongman Jeon

E-mail: kjeon@skku.edu

(C) The Korean Society for Transplantation

This is an Open Access article distributed under the terms of the Creative Commons Attribution Non-Commercial License (http://creativecommons.org/licenses/by-nc/4.0/) which permits unrestricted non-commercial use, distribution, and reproduction in any medium, provided the original work is properly cited. 